Open Access

\title{
Tuberous sclerosis complex neuropathology requires glutamate-cysteine ligase
}

\author{
Anna R. Malik, Ewa Liszewska', Agnieszka Skalecka', Malgorzata Urbanska', Anand M. lyer², Lukasz J. Swiech', \\ Malgorzata Perycz' ${ }^{1}$ Kamil Parobczak', Patrycja Pietruszka', Malgorzata M. Zarebska', Matylda Macias', \\ Katarzyna Kotulska ${ }^{5}$, Julita Borkowska ${ }^{5}$, Wieslawa Grajkowska ${ }^{5}$, Magdalena E. Tyburczy ${ }^{6}$, Sergiusz Jozwiak \\ David J. Kwiatkowski ${ }^{6}$, Eleonora Aronica ${ }^{2,3,4}$ and Jacek Jaworski ${ }^{{ }^{*}}$
}

\begin{abstract}
Introduction: Tuberous sclerosis complex (TSC) is a genetic disease resulting from mutation in TSC1 or TSC2 and subsequent hyperactivation of mammalian Target of Rapamycin (mTOR). Common TSC features include brain lesions, such as cortical tubers and subependymal giant cell astrocytomas (SEGAs). However, the current treatment with mTOR inhibitors has critical limitations. We aimed to identify new targets for TSC pharmacotherapy.

Results: The results of our shRNA screen point to glutamate-cysteine ligase catalytic subunit (GCLC), a key enzyme in glutathione synthesis, as a contributor to TSC-related phenotype. GCLC inhibition increased cellular stress and reduced mTOR hyperactivity in TSC2-depleted neurons and SEGA-derived cells. Moreover, patients' brain tubers showed elevated GCLC and stress markers expression. Finally, GCLC inhibition led to growth arrest and death of SEGA-derived cells.
\end{abstract}

Conclusions: We describe GCLC as a part of redox adaptation in TSC, needed for overgrowth and survival of mutant cells, and provide a potential novel target for SEGA treatment.

Keywords: Tuberous Sclerosis Complex, Glutamate-cysteine ligase, Cellular stress, Brain tumors, Cell death

\section{Introduction}

Tuberous sclerosis complex (TSC) is a common neurocutaneous disease, inherited in an autosomal dominant pattern, affecting 1:6,000 live births [29]. It is caused by mutations in either of two genes encoding components of the TSC protein complex: TSC1 (hamartin) and TSC2 (tuberin). Disrupted TSC complex function results in overactivation of mammalian Target of Rapamycin complex 1 (mTORC1), an important regulator of cell metabolism [26]. mTORC1 overactivation is believed to be the main cause of the pathological overgrowth of cells and development of tubers in different tissues, including the brain. Patients develop 3 types of brain lesions: subependymal nodules, cortical tubers and subependymal giant cell astrocytomas (SEGAs) of glio-neuronal origin $[4,27]$. The last two contribute to TSC clinical

\footnotetext{
* Correspondence: jaworski@iimcb.gov.pl

'Laboratory of Molecular and Cellular Neurobiology, International Institute of Molecular and Cell Biology, Warsaw 02-109, Poland

Full list of author information is available at the end of the article
}

symptoms-cortical tubers are considered as epileptogenic [18], while growth of SEGA is commonly associated with the development of hydrocephalus and can lead to severe complications, including death [30]. Extensive research concerning tuberous sclerosis complex has led to a breakthrough in the treatment of this disease, enabling pharmacotherapy with mTOR inhibitors $[20,30]$. mTOR inhibitors reportedly decrease SEGA size, and everolimus was recently approved by the FDA and EMA for TSC-associated SEGAs [34]. Moreover, many reports indicate that mTOR inhibition may alleviate epilepsy severity in TSC patients $[21,44]$. However, withdrawal of mTOR inhibitor is associated with rapid tumor regrowth, and mTOR inhibitors use is burdened with significant adverse events, including infections, mouth ulcers, and hyperlipidemia [38]. In some cases, use of mTOR inhibitors can even lead to life-threatening complications, including sepsis and death [41]. Thus, the development of a more efficient and safer drug is of utmost importance. 
Therefore, in this study, we aimed to provide new potential drug targets in TSC pharmacotherapy by identifying proteins crucial for the pathological overgrowth typical of TSC. We report results of an shRNA screen, in which we silenced expression of genes potentially linked to TSC pathology or mTOR signaling. We identified several proteins that possibly contribute to TSC-related phenotype. Among them, we focus on the role of glutamate-cysteine ligase catalytic subunit (GCLC), a protein important for the control of oxidative stress levels. We show that GCLC inhibition increases cellular stress and reduces mTOR hyperactivity in TSC2-depleted neurons and SEGA-derived cells. Moreover, we provide evidence that this protein might be a potential target in the pharmacotherapy of TSC.

\section{Materials and methods}

\section{Drugs and antibodies}

All drugs and antibodies used for the study are described in Supplementary materials and methods (Additional file 1).

\section{DNA constructs}

pCx EGFP-N1, pSuper and $\beta$-actin-GFP [19] plasmids have been described previously $[2,3,19]$. The sequences encoding shRNAs used in the screen were cloned into the pSuper vector and are listed in Additional file 2: Table S1. mRNA sequences targeted in TSC2 and GCLC as well as cloning strategy for TSC2 and its shRNAresistant mutants is described in Supplementary materials and methods (Additional file 1).

\section{Neuronal cultures, transfection, drug treatments, and immunofluorescence}

The animals used to obtain neurons for tissue cultures were sacrificed according to the protocols approved by the First Ethical Committee in Warsaw, Poland (permission \#75/ 2010), which are in compliance with the European Community Council Directive (86/609/EEC). Primary cortical cultures were prepared from embryonic day 19 (E19) rat brains as described previously [25]. For the purpose of immunofluorescence and morphometric analysis, neurons were transfected on Day 6 in vitro (DIV6) with Lipofectamine2000 as described earlier [35]. Twenty nanomolar rapamycin or $10 \mu \mathrm{M} \mathrm{L}$-BSO were added to the medium $2 \mathrm{~h}$ after transfection and the medium was not changed until the end of the experiment. Neurons were fixed with $4 \%$ paraformaldehyde (PFA) and $4 \%$ sucrose in PBS at DIV10. Immunofluorescent staining was performed as described recently [25].

When a highly efficient gene transfer was required (Western blot), plasmids were introduced to freshly isolated neurons (DIV0) using the AMAXA nucleofection procedure (Lonza, Basel, Switzerland) as described recently [25]. For GCLC inhibition in nucleofected cells,
$10 \mu \mathrm{M} \mathrm{L}$-BSO was added to the medium $18 \mathrm{~h}$ post nucleofection. Neurons were lysed $30 \mathrm{~h}$ later.

\section{SEGA-derived cell culture and treatment}

The study was approved by The Ethics Board at the Children's Memorial Health Institute, Warsaw, Poland. The samples of patients' SEGAs were analyzed after written consent was obtained from their parents. Patients were diagnosed as having TSC according to Roach's criteria. The patients presented with acute hydrocephalus and were operated after large SEGAs were revealed in brain MRI (Additional file 3: Figure S3a). Freshly resected SEGA samples from two patients were cut into small pieces and trypsynized for $1 \mathrm{~h}$ at $37{ }^{\circ} \mathrm{C}$. After trypsinization the tissue fragments were dispersed with a pipette to small clumps or single cells. The obtained cell suspension was centrifuged and the pellet was suspended in DMEM $4.5 \mathrm{~g} / \mathrm{l}$ glucose supplemented with $5 \%$ fetal bovine serum (FBS; Gibco, Karlsruhe, Germany) and antibiotics (100 U/ml penicillin, $100 \mu \mathrm{g} / \mathrm{ml}$ streptomycin; Sigma, St. Louis, MO). Cells were maintained for about 2 weeks until they reached confluence and were used for experiments.

For live imaging experiments, cells were plated on gelatin-coated $\mu$-Slide VI 0.4 plates (Ibidi, Planegg, Germany). During pharmacological treatment, the medium was changed every second day and the drugs were used as follows: U0126 $(20 \mu \mathrm{M})$, rapamycin $(20 \mathrm{nM})$, L-BSO $(20$ or $100 \mu \mathrm{M})$.

\section{COS-7 cell culture and transfection}

COS-7 cells (ATCC) were cultured in DMEM supplemented with $10 \% \mathrm{FBS}$ and antibiotics. Cells were transfected using Lipofectamine2000 (Invitrogen, Carlsbad, CA) according to manufacturer protocol.

\section{shRNA library screen}

Target genes covered by the shRNA library are listed in Supplementary materials and methods (Additional file 1). The majority of targets was selected based on published data $[5,6,45]$; for more detailed description see Supplementary materials and methods (Additional file 1). Whenever possible, 3 shRNAs were designed against given mRNA and shRNAs coding sequences and cloned into the pSuper vector. However, in some cases, only two shRNAs could be designed.

In screening experiments, cortical neurons were transfected on Day 6 in vitro (DIV6) with TSC2sh together with pools of pSuper plasmids that encoded shRNAs targeting a given gene and $\beta$-actin-GFP. Each culture plate contained 3 control variants: (i) transfected with pSuper/ $\beta$-actin-GFP, (ii) transfected with TSC2sh/pSuper/ $\beta$-actin-GFP, and (iii) transfected with TSC2sh/pSuper/ $\beta$ actin-GFP and treated with $20 \mathrm{nM}$ rapamycin. Four days 
after transfection, the neurons were fixed and cell images were acquired. To avoid variability caused by differences between cultures, the area of neuron soma was quantified as a percentage of the mean value obtained for neurons in $\mathrm{pSuper} / \beta$-actin-GFP control variant from the same experimental plate. Two independent screening experiments were performed, and the mean value was calculated from both of them for each shRNA pool.

\section{In vivo electroporation in neonates}

All the procedures were approved by the First Ethical Committee in Warsaw, Poland (permission \#569/2014), which is in compliance with the European Community Council Directive (86/609/EEC). Neonates (P0; Wistar, both sexes) were anesthetized by hypothermia and the plasmid solution was injected to the right lateral ventricle. Next, the animals were subjected to electrical pulses. Electroporated animals were warmed on a heating pad for several minutes before being returned to the mother. For detailed electroporation protocol, rat tissue preparation and staining procedures see Supplementary materials and methods (Additional file 1).

\section{Human tissue samples}

The cases included in this study were obtained from the archives of the departments of neuropathology of the Academic Medical Center (University of Amsterdam) and the University Medical Center in Utrecht (UMCU). We examined 10 surgical specimens, 5 cortical tubers (male/female: $3 / 2$; mean age at surgery: 16.8 years, range: 10-23), and 5 subependymal giant-cell astrocytomas (SEGA; male/female: 3/2; mean age at surgery: 12 years, range: 1-23) from patients undergoing epilepsy surgery or surgery for obstructive hydrocephalus. Informed consent was obtained for the use of brain tissue and for access to medical records for research purposes. Tissue was obtained and used in a manner compliant with the Declaration of Helsinki. For the SEGA, we used the WHO classification of tumors of the central nervous system [24]. All patients fulfilled the diagnostic criteria for TSC [11]. All patients included had TSC2 mutations. Normal-appearing control cortex was obtained at autopsy from 5 age-matched patients without history of seizures or other neurological diseases. Autopsied brain tissues from patients with Alzheimer's disease (AD) and trauma (as well as skin specimens) were also examined (as positive controls for immunohistochemical analysis; $\mathrm{AD}$ and trauma specimens (for HO-1 and Hsp70) and skin specimens (for GCLC)). All autopsies were performed within $24 \mathrm{~h}$ after death. Histologically normal temporal neocortex (without evidence of significant neuronal loss, gliosis, or malformation) from two patients undergoing extensive surgical resection of the mesial structures for the treatment of medically intractable complex partial epilepsy was also used for immunocytochemical analysis. For human tissue preparation and staining procedures, see Supplementary materials and methods (Additional file 1).

\section{Image analysis and quantification}

Olympus Cell ${ }^{\wedge} \mathrm{R}$ station was used to obtain neuron images in screening experiments and for live imaging of SEGA-derived cells. Confocal images of immunofluorescently stained cortical neurons and rat brain sections were obtained with a Zeiss LSM710NLO microscope. The morphometric analysis of neuron soma area and immunofluorescence intensity analysis were performed via MetaMorph image analysis software (Universal Imaging Corporation, Downingtown, PA) with Integrated Morphometry Analysis and Region Measurements functions, respectively. For the analysis of SEGA-derived cells' area, ImageJ software was used. For 3D reconstruction and morphometric analysis of GFP positive cells from rat brain slices, Imaris software (Bitplane, Zurich, Switzerland) was used.

\section{Statistical analysis}

The data concerning neurons were obtained from three batches of cells, with the exception of the screening experiment that was performed on two batches of neurons. SEGA live imaging was performed twice for each of two independent SEGA-derived cultures. For animal studies, we reduced the number of animals to the minimum required for reliable statistics. Animals were randomly allocated to experimental groups and no blinding was done. The specimens were coded for microscopy analysis. The statistical analyses were performed using Prism Software (GraphPad, San Diego, CA) and Kruskal-Wallis test followed by Dunn's post hoc test, or two-way ANOVA depending on the type of data analyzed. In the latter case variance was similar between compared groups. The number of values (analyzed cells) and animals in experimental groups is provided in Supplementary materials and methods (Additional file 1).

\section{Results}

Screening experiments reveal new proteins indispensable for abnormal growth of TSC2-depleted neurons

One characteristic feature of TSC is the presence of enlarged dysmorphic neurons and giant cells in cortical tubers [13]. This aberrant cell size increase is thought to be due to loss of either TSC1 or TSC2 and mTORC1 overactivation [12, 39]. To enable screening for the genes and proteins required for the mTORC1dependent cell size increase, we first modeled aberrant neuronal growth in vitro. Utilizing plasmid-based shRNA expression [3], we efficiently depleted cultured rat cortical neurons of TSC2 (Additional file 3: Figure S1a) using either one of two shRNAs (TSC2sh\#1, TSC2sh\#2). 
When introduced to neurons at Day 6 in vitro (DIV6), these shRNAs effectively increased (i) levels of ribosomal S6 protein phosphorylated at Ser235/236 (P-S6) and (ii) neuronal soma size (Fig. 1a). Both observations are consistent with the data from TSC patients [36], TSCmodel mouse brain tissue [12, 43], and in vitro TSC models $[28,39]$. Importantly, both the increase in P-S6 levels and cell soma size were blocked by treatment with

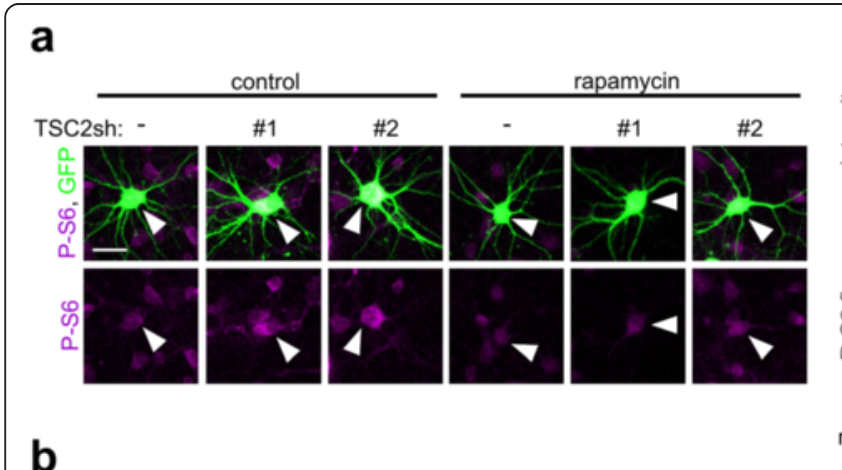

b

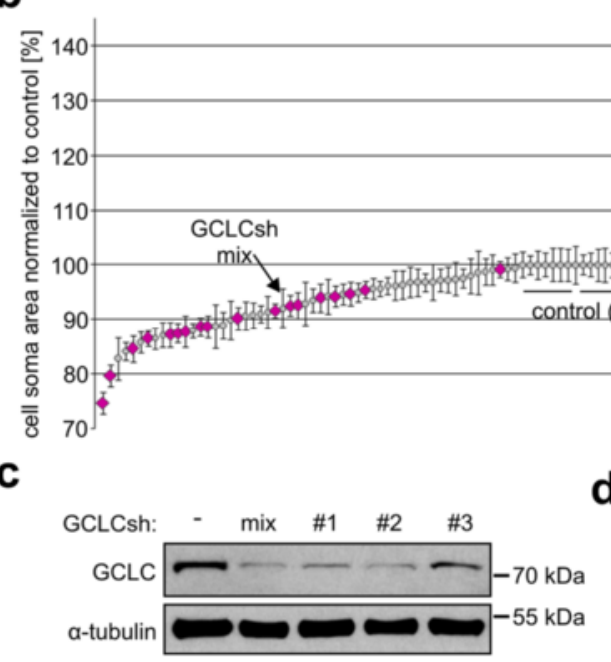

e

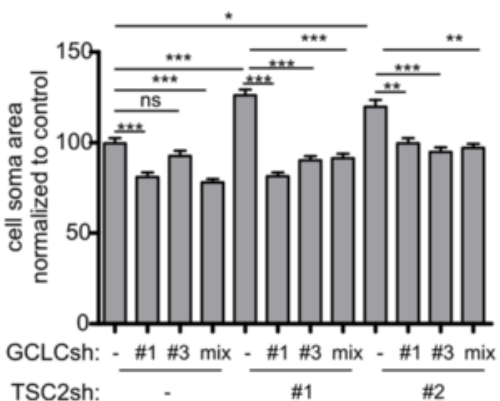

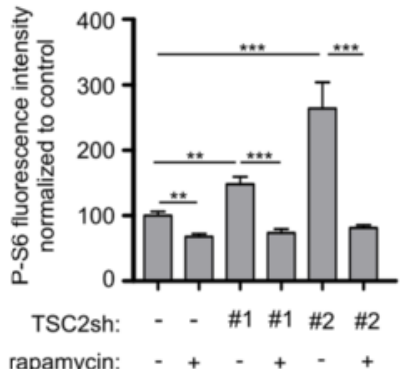

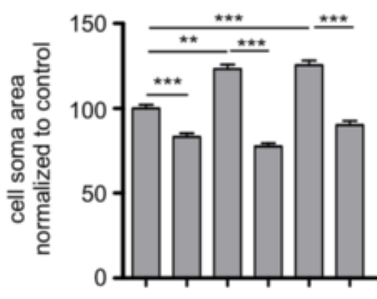

TSC2sh: - - \#1 \#1 \#2 \#2

rapamycin: -+++-+ rapamycin:

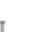


an mTOR inhibitor (20 nM rapamycin) (Fig. 1a). The specificity of both TSC2sh was confirmed by "rescue" experiments, in which neuronal overgrowth was prevented by co-transfecting neurons with TSC2sh and a plasmid encoding TSC2 mRNA resistant to shRNA (Additional file 3: Figure S1b and S1c).

Having validated our in vitro model of mTORdependent neuronal hypertrophy, we performed screening experiments to identify genes and proteins necessary for the aberrant cell size increase. To this end, we used a library of shRNAs designed to target mRNAs encoding 147 proteins linked to mTOR signaling pathway or TSC pathology in past studies (see Supplementary materials and methods (Additional file 1) for detailed library description and the list of targeted genes and Additional file 2: Table S1 for shRNAs sequences). shRNAs targeting given mRNA were transfected to cortical neurons as pools to ensure effective knockdown. The pools of shRNAs were co-transfected to DIV6 neurons with TSC2sh\#1 and plasmid encoding GFP, for visualization of transfected cell morphology. As a control, we used neurons transfected with (i) empty pSuper, (ii) pSuper and TSC2sh\#1, and (iii) pSuper and TSC2sh\#1, treated with $20 \mathrm{nM}$ rapamycin. Analysis of the cell somas of transfected neurons 4 days post transfection revealed that the majority of tested shRNAs had no effect on TSC2sh\#1-induced hypertrophy. However, 30 shRNA pools caused a significant decrease in the neuronal soma area of TSC2-depleted cells (Additional file 3: Figure S1b and Table S2). Twenty-three of these genes were confirmed in follow-up experiments using TSC2sh\#2 instead of TSC2\#sh1 (Additional file 3: Table S2).
TSC-related neuronal hypertrophy depends on GCLC

We subsequently decided to focus on GCLC, a first rate limiting enzyme of glutathione synthesis that was previously identified in a yeast study as potentially linked to TOR signaling [5]. GCLC plays a crucial role in protecting cells from oxidative stress and damage caused by reactive oxygen species (ROS). There is also evidence indicating increased cellular stress and ROS levels in TSC pathology [28].

To verify the results of screening experiments, we tested the specificity of GCLC shRNAs and its phenotypic effects. Indeed, all three GCLCsh individually and as a pool efficiently reduced levels of GCLC in nucleofected cortical neurons cultured in vitro (Fig. 1c). We next tested effects of single shRNAs on the phenotype of the model hypertrophic neurons and confirmed that both GCLCsh\#1 and GCLCsh\#3, similar to the GCLCsh pool, reversed the hypertrophic phenotype induced by TSC2 depletion with either TSC2sh\#1 or TSC2sh\#2 (Fig. 1d and 1e). GCLCsh\#2 appeared to have nonspecific toxic effects when transfected to DIV6 neurons, and therefore was not studied further.

We next examined whether GCLC is important for the hypertrophy of TSC2-depleted neurons in vivo. For this purpose, we performed in vivo electroporation of shRNAs encoding plasmids into proliferating neuronal precursors in the subventricular zone (SVZ) of newly born rodents (Fig. 2a), as described previously [8, 22]. Six days after electroporation, the TSC2sh\#2-electroporated neuroprogenitors in the rostral migratory stream had a significantly higher volume when compared to the cells of control rats (Fig. 2b-2d). Consistent with our

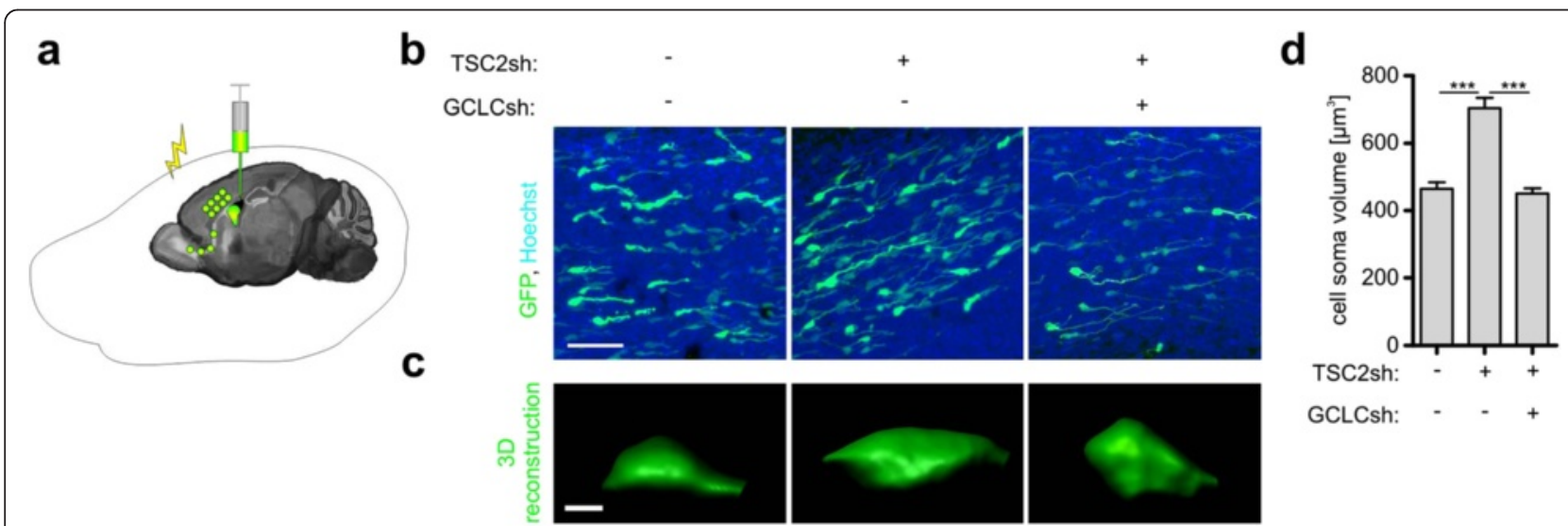

Fig. 2 GCLCsh blocks aberrant growth in TSC in vivo model. a Scheme of in vivo electroporation. b Representative images of cells in rostral migratory stream obtained in in vivo experiments. Newborn rats were electroporated with pSuper vector or TSC2sh\#2 together with pSuper or GCLCsh\#1. Plasmid encoding GFP was used to visualize modified cells. Scale bar: $50 \mu \mathrm{m}$. c Exemplary 3D reconstructions of GFP-positive cells in rostral migratory stream. Scale bar: $3 \mu \mathrm{m}$. d Quantification of cell soma volume of cells migrating in the rostral migratory stream. Plot represents mean +/-SEM. ${ }^{* * *} \mathrm{p}<0.001$ in Kruskal-Wallis with Dunn's post-hoc test. Sample sizes for experimental groups are provided in Supplementary materials and methods (Additional file 1) 
observations from cultured neurons, co-electroporation of GCLCsh with TSC2sh blocked the size increase of TSC2-deficient cells.

Furthermore, we verified the importance of GCLC for aberrant growth of TSC2-defficient neurons using a specific inhibitor of GCLC catalytic activity, L-Buthioninesulfoximine (L-BSO) [14]. Neuronal soma enlargement was fully blocked by the addition of L-BSO $(10 \mu \mathrm{M})$ to the cultured neurons transfected with TSC2sh (either \#1 or \#2), supporting our finding that GCLC is necessary for TSC-related aberrant growth (Fig. 3a).

\section{GCLC inhibition increases cellular stress and reduces mTOR activation in TSC2-depleted neurons}

Previous work showed that knocking out or down TSC2 elevates cellular stress [28,32] and eventually leads to apoptotic death in neurons [28]. Yet, TSC-lacking brain cells survive in both mouse models and TSC patients' brain lesions, suggesting their adaptation to such stress. We thus hypothesized that if GCLC contributes to this adaptation, blocking its activity in TSC2-deficient cells should perturb the redox balance. Consistent with previous findings, TSC2 knockdown neurons showed higher levels of oxidative stress markers-heme oxygenase 1 (HO-1) and heat shock protein 70 (Hsp70)-compared to control cells (Fig. 3b). Importantly, inhibiting GCLC activity with L-BSO further increased $\mathrm{HO}-1$ and $\mathrm{Hsp} 70$ in TSC2-deficient cells but did not lead to visible induction of those markers in control neurons (Fig. 3b). We also note that GCLC level is higher in TSC2-depleted cells and, in contrast to control cells, cannot be further increased upon L-BSO treatment (Fig. 3b). As GCLC mitigates oxidative stress, its elevated expression is likely an adaptive response to an increase in ROS in TSC2 knockdown neurons. Inhibition of GCLC seems to disturb this adaptation, increases cellular stress, and prevents aberrant cell size increase. Importantly, GCLC inhibition decreases mTORC1 activity reflected by P-S6 levels in TSC2-depleted neurons but not in control cells (Fig. 3b), which could explain the inhibition of aberrant growth.

TSC patients' brain lesions show increased levels of stress markers and GCLC

Assuming that GCLC-dependent oxidative stress adaptation is necessary to develop TSC-related cellular hypertrophy, we should observe increased stress markers and GCLC also in cortical tubers in patients' tissue. To test this, we immunostained brain sections from individuals affected with TSC for HO-1 and Hsp70 as well as for GCLC (Fig. 4, see also Additional file 3: Figure S2 for additional controls). Consistent with the conclusions from published data [28] and our in vitro studies, we observed the presence of those proteins in dysmorphic neurons and the giant cells of cortical tubers, but not in

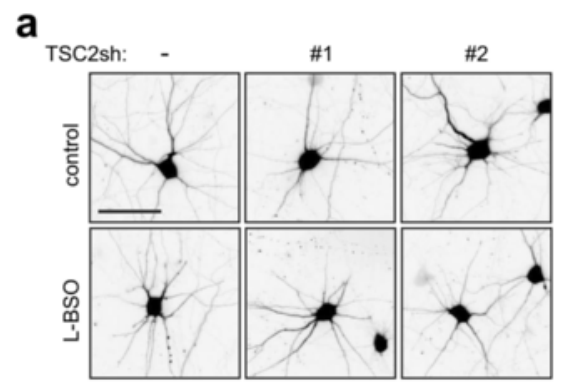

b
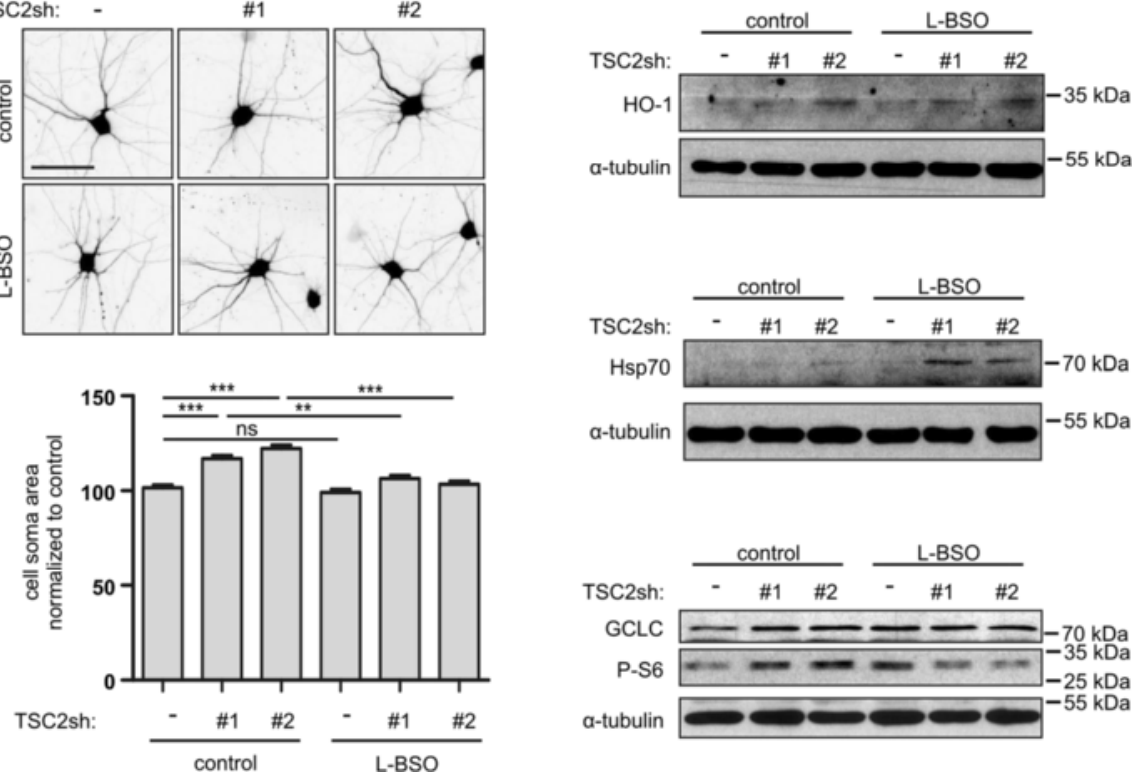

Fig. 3 GCLC inhibitor L-BSO blocks aberrant growth, induces stress response and inhibits mTORC1 in TSC2 depleted neurons. a Representative images and neuron soma area of TSC2-depleted and control neurons treated with $10 \mu \mathrm{M}$ L-BSO. Scale bar: $50 \mu \mathrm{m}$. Plot represents mean + -SEM. ns- not significant, ${ }^{* *} p<0.01,{ }^{* *} p<0.001$ in Kruskal-Wallis with Dunn's post-hoc test. Sample sizes for experimental groups are provided in Supplementary materials and methods (Additional file 1). b Western blot analysis of HO-1, Hsp70, GCLC and mTORC1 activity marker, P-S6, levels in TSC2-depleted and control neurons after L-BSO treatment. a-tubulin is shown as a loading control 


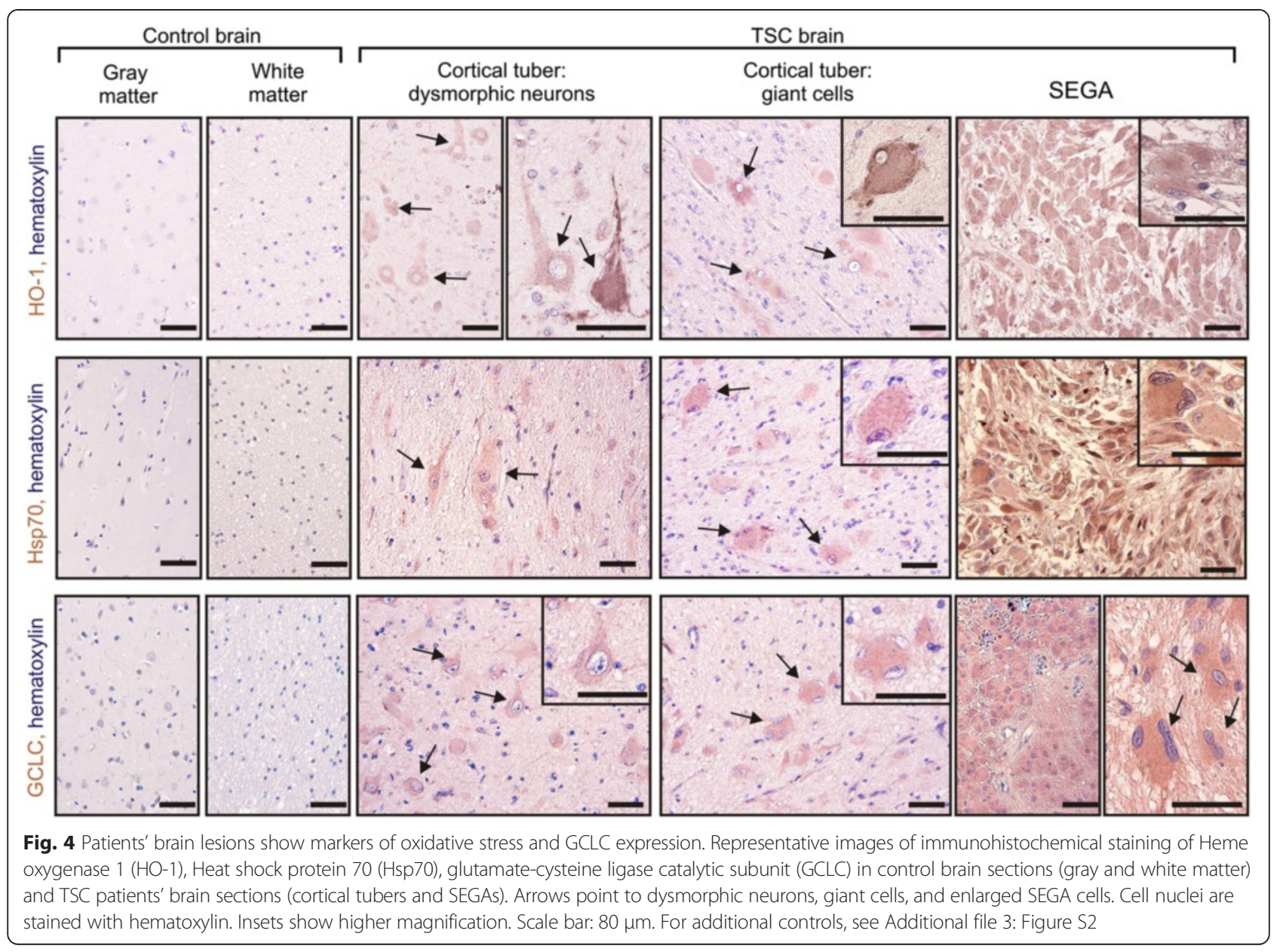

control brain tissue from individuals not affected with TSC, nor in surrounding normal-appearing cells (Fig. 4). Intriguingly, similar results were obtained for enlarged SEGA cells as compared to control brain tissue (Fig. 4), suggesting that the hypertrophy of cells in different types of brain TSC lesions may depend on GCLC.

\section{GCLC inhibition affects SEGA-derived cells growth, proliferation, and survival}

Since SEGAs are constantly growing lesions that often require surgical resection or treatment with mTORC1 inhibitors, we examined the effects of GCLC inhibition on the growth of cultured SEGA cells derived at the time of surgical resection from two TSC patients (Additional file 3: Figure S3a). To circumvent the heterogeneity of the cells observed in these cultures, we used live imaging of SEGAderived cells during treatment with L-BSO (Fig. 5). This approach enabled reliable quantification of cell size increase in comparison to initial cell area and allowed us to exclude from analysis cells that divided or died over this time.

L-BSO added to the culture media, at either 20 or $100 \mu \mathrm{M}$, significantly slowed down the growth of two SEGA-derived lines (Fig. 5a). A similar effect, although less potent than that of a higher L-BSO dose, was obtained upon rapamycin or rapamycin/U0126 (extracellular signalregulated kinase inhibitor) treatment, as described previously, on cultured SEGA-derived cells [42]. On the other hand, lower dose of L-BSO combined with rapamycin slowed down growth of tested cells similar to the $100 \mu \mathrm{M}$ L-BSO (Fig 5a).

Of note, L-BSO treatment in both cell lines also induced expression of cellular stress markers Hsp70 and $\mathrm{HO}-1$, as well as Nrf2, a transcription factor coordinating expression of several oxidative stress-inducible genes, including HO-1 [16]. Moreover, as in case of TSC2-depleted neurons, L-BSO treatment lead to decrease in P-S6 levels (Fig. 5b; see also Additional file 3: Figure S4 for WB results quantification). It should be noted however, that Ser 235/236 S6 phosphorylation could also decrease due to a drop in extracellular-signal-regulated kinases (ERKs) activity in response to L-BSO. Therefore, we checked levels of P-ERK1/2 (Thr 202/Tyr 204) in L-BSO-treated cells and could not detect any changes over time (Additional file 3 : Figure S5). In contrast, we noticed a reduced phosphorylation of Thr 389 of p70S6K, a direct mTORC1 target (Additional file 3: Figure S5). This supports our initial 

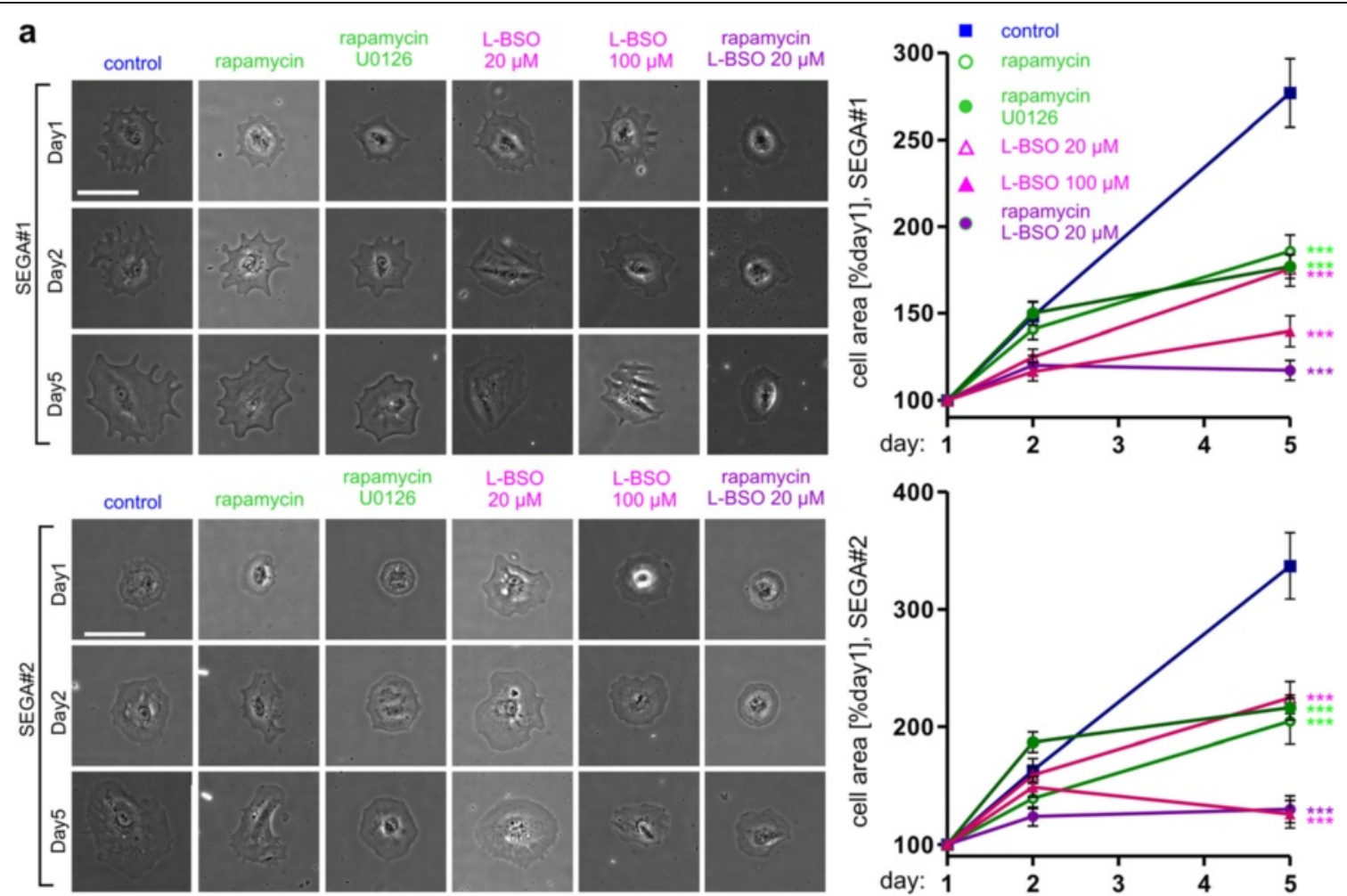

$20 \mu \mathrm{M}$

$100 \mu \mathrm{M}$

rapamycin

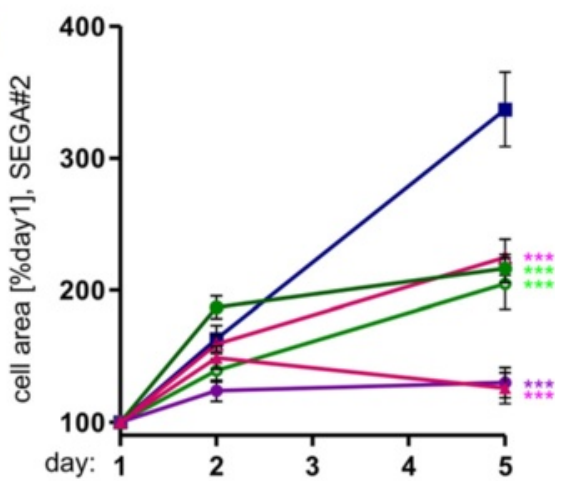

SEGA\#1
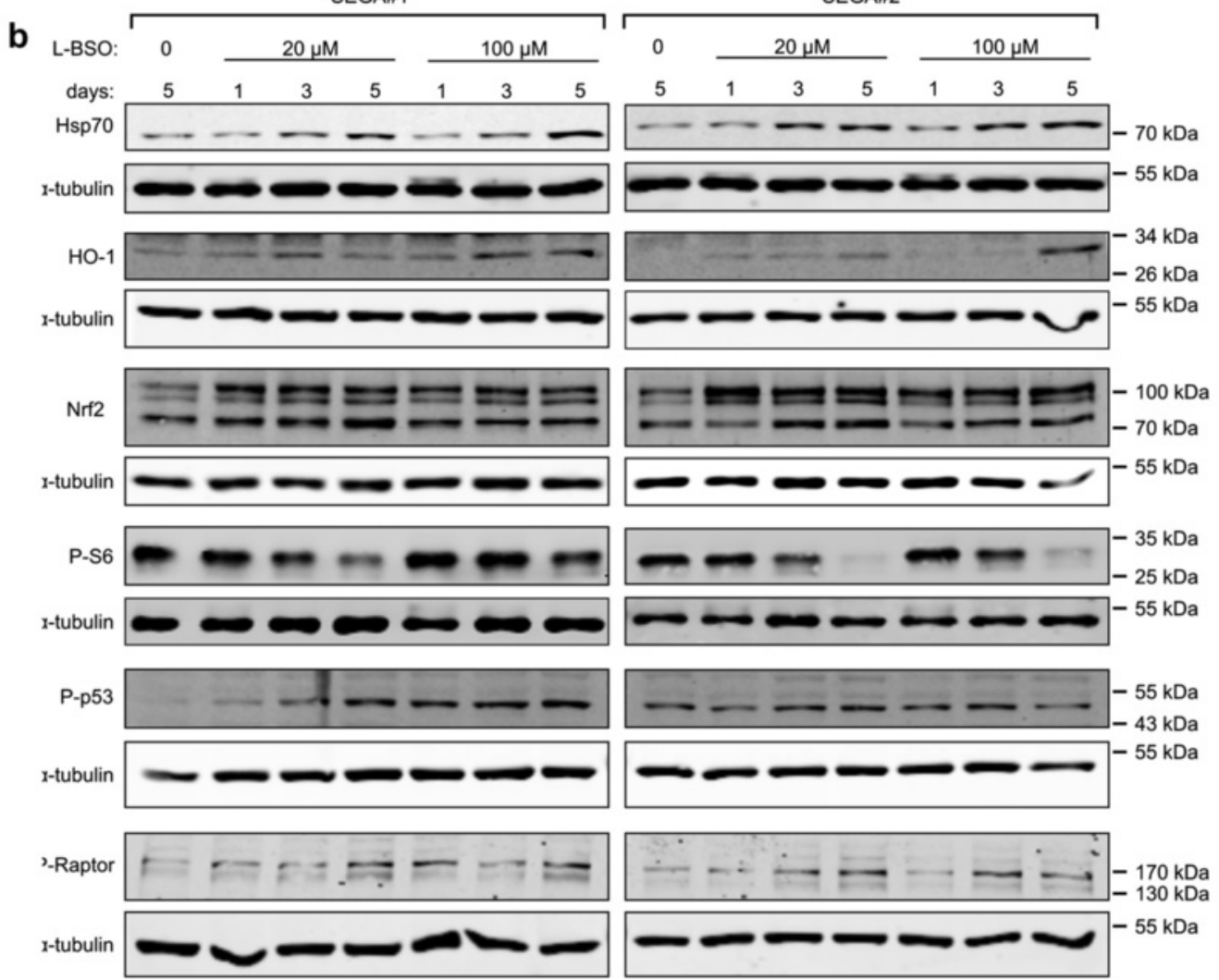

Fig. 5 (See legend on next page.) 
(See figure on previous page.)

Fig. 5 GCLC inhibition blocks growth of SEGA-derived cells and causes cellular stress, and mTORC1 inhibition. a Representative images (left) and results of cell surface area analysis (right) of SEGA-derived cells imaged 3 times during a 5 day-treatment. SEGA\#1 and SEGA\#2-derived cells were treated with $20 \mathrm{nM}$ rapamycin, $20 \mathrm{nM}$ rapamycin with $20 \mu \mathrm{M}$ U0126 or $20 \mu \mathrm{M}$ L-BSO, and 20 or $100 \mu \mathrm{M}$ L-BSO. Scale bar: $100 \mu \mathrm{m}$. The plots represent mean +/- SEM from two independent experiments. ${ }^{* *} \mathrm{p}<0.001$ in two-way ANOVA compared to control treated with DMSO. Sample sizes for experimental groups are provided in Supplementary materials and methods (Additional file 1). b Western blot analysis of stress markers (Hsp70, HO-1), Nrf2, mTORC1 activity marker P-S6, genotoxic stress marker P-p53 and P-Raptor in SEGA-derived cells lysed after a 1-, 3- or 5-day treatment with 20 or $100 \mu \mathrm{M}$ L-BSO. a-tubulin is shown as a loading control

conclusion that L-BSO gradually decreases mTORC1 activity in SEGA-derived cells. We hypothesized that the observed mTORC1 inhibition is an effect of ROS-induced DNA damage. Indeed, supporting such mechanism, SEGA-derived cells treated with L-BSO show progressive increase in $\mathrm{p} 53$ phosphorylation (P-p53, Ser15), which is a hallmark of DNA damage response [37] (Fig. 5b; see also Additional file 3: Figure S4 for WB results quantification). We also observed parallel increase in inhibitory phosphorylation of Raptor (P-Raptor, Ser792), a key component of mTORC1 (Fig. 5b; see also Additional file 3: Figure S4 for WB results quantification). P-p53 has been shown to activate AMPK $[7,10]$, which in turn phosphorylates Raptor and inhibits mTORC1 [15].

It is well established that genotoxic stress may affect cell proliferation and survival. To address this issue, we examined the effects of longer periods of L-BSO treatment on SEGA-derived cell fates. For this purpose, we performed live imaging for a total of 8 days. For quantification, we divided cells present at day 1 into 3 classes: cells that finally gave rise to one cell by Day 8 (in most cases neither divided nor died; class A), cells that gave rise to two or more cells (in most cases divided at least once; class B), and those that died (class C) (Fig. 6a, see also Additional file 3: Figure S3b and S3c for a more detailed description of classes). In response to prolonged L-BSO treatment, the two SEGA-derived cell lines both showed marked reduction in cell division, and increased numbers of cells were lost. These effects were more dramatic compared to treatment with either rapamycin alone or combined with U0126 (Fig. 6b). However, $20 \mu \mathrm{M}$ L-BSO-rapamycin combined treatment gave effects comparable to higher L-BSO dose (Fig. 6b). Moreover, L-BSO treatment induced cleavage of Poly(ADP-ribose) polymerase 1 (PARP-1; Fig. 6c), confirming SEGA-derived cells death.

In conclusion, we propose that mechanisms underlying L-BSO effects on TSC cells involve induction of redox imbalance, oxidative DNA damage and subsequent increase in P-p53. These events lead consequently to growth arrest due to AMPK-P-Raptor-dependent inhibition of mTORC1 and genotoxic cell death.

\section{Discussion}

Our data clearly established the requirement of GCLC for the development of TSC-related phenotype. Findings from in vitro and in vivo models, as well as study of SEGA-derived cell growth, point to GCLC inhibition as a potential strategy in TSC treatment. Moreover, we observed substantial induction of cellular stress markers and GCLC in TSC patients' brain tubers, which supports the clinical relevance of our findings.

The primary finding of our study is that GCLC contributes to the hypertrophy and survival of TSC2-deficient cells. We describe increased cellular stress marker expression and mTORC1 inhibition upon L-BSO treatment, both in case of TSC2 neuronal knockdown and SEGA-derived cells. Mechanisms of this inhibition most likely involve genotoxic stress-dependent p53 phosphorylation and subsequent inactivation of mTORC1 due to AMPK-dependent phosphorylation of Raptor. Importantly, mTORC1 inhibition upon L-BSO treatment was observed exclusively in TSC2-deficient cells, which may have important clinical implications.

Our data suggest that GCLC provides protection against elevated ROS level in TSC2-deficient cells. Redox adaptation has also been described for various cancer cells [40]. The signatures of this phenomenon include induction of ROS scavenging enzymes expression and increased sensitivity to the depletion of glutathione. In a similar vein, it has been demonstrated that expression of Hif $1 \alpha$, known to be a part of antioxidant defense in response to ROS, is highly elevated in TSC1-deficient cells $[9,33]$. Herein, we provide evidence for increased GCLC levels, suggesting that this ROS scavenging systems is also upregulated. Targeting redox homeostasis or increasing cellular stress is considered a supplemental therapy for many cancers [40]. Previously, increasing ER stress proved efficient in boosting selective cell death in TSC-deficient cells, and was therefore suggested as a potential therapy $[28,32]$. In this paper, we demonstrate that another approach, namely, targeting the synthesis of ROS scavengers, is effective in preventing cellular hypertrophy as well as inducing the loss of cells with disturbed TSC, likely due to mTORC1 inhibition and genotoxic DNA damage, respectively. Of note, unlike ER stress inductor tunicamycin, L-BSO has some synergistic effects with rapamycin, which might be beneficial for TSC pharmacotherapy. Our observations are also in line with recent work from Blenis lab, showing that combined ER stress induction and glutathione depletion 


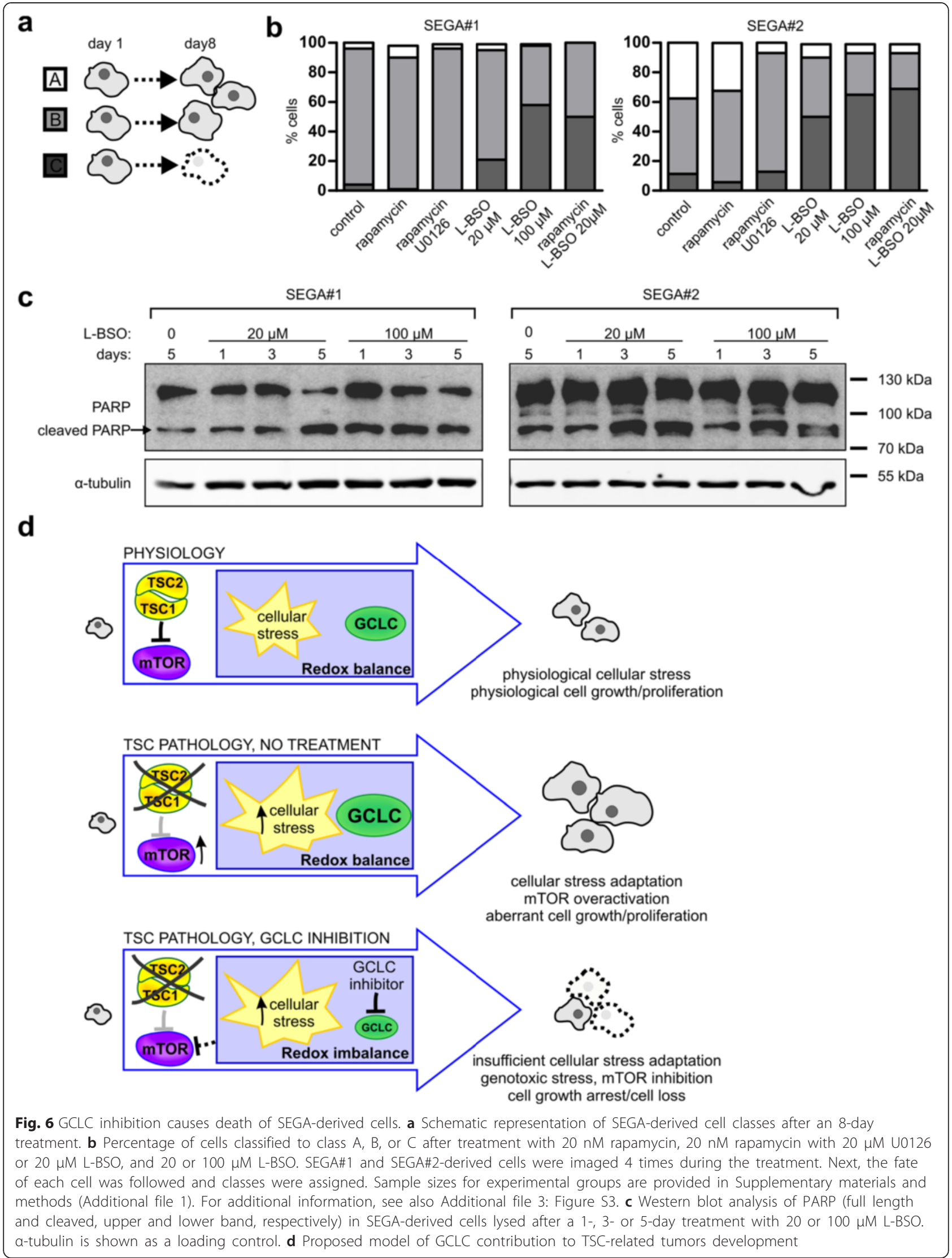


leads to death of TSC2 knockout fibroblasts cultured in vitro and regression of TSC2-deficient xenograft tumors development [23]. This finding strongly corroborates our conclusion that inhibition of GCLC can be considered as a new, strategy for treatment of different types of TSC-related tumors. Moreover synergistic effects of GCLC inhibition with rapamycin treatment would allow combined therapy.

Our results thus suggest that GCLC inhibition could be a novel strategy in the pharmacotherapy of TSCrelated brain tubers. However, the consequences of systemic GCLC inhibition remain underinvestigated. For example, BSO toxicity was shown in rats [17]. Nevertheless, BSO was tested in phase I clinical trial for treatment of refractory malignancies with the conclusion that it could be safely administered to patients [1]. Therefore, in our opinion, this line of research is promising, since currently available pharmacotherapy of TSC-related brain tubers, based on mTOR inhibitors, has important limitations. Although certainly beneficial for patients, the treatment does not cause persistent eradication of SEGA tubers and therefore needs to be continued for longer periods of time [31]. In addition, although in our study rapamycin treatment reduces SEGA-derived cell growth, it does not lead to cell loss. On the contrary, L-BSO alone or at lower doses in combination with rapamycin (not shown) caused SEGA-derived cell death. If the same results are obtained in patient treatment, pharmacotherapy could perhaps be shortened, which would be a great advantage over mTOR inhibitors, known to severely impair vital cellular processes also in healthy cells. In summary, we identified GCLC as an important player in TSC-related hypertrophy and a potential target for the future pharmacotherapy of SEGA.

\section{Conclusions}

In this study, we aimed to provide new potential drug targets in TSC pharmacotherapy by identifying proteins crucial for the pathological overgrowth typical of TSC. Consequently, using in vitro and in vivo rodent models, as well as SEGA-derived cells cultured in vitro we describe GCLC as a part of redox adaptation in TSC, needed for overgrowth and survival of mutant cells. Inhibition of GCLC with L-BSO was able to prevent TSC related cell hypertrophy and induce mutant cell death, suggesting GCLC inhibition as a potential strategy in TSC treatment. We propose that mechanisms underlying effects of L-BSO on TSC cells involve induction of redox imbalance, oxidative DNA damage and subsequent increase in P-p53. These events lead consequently to growth arrest due to AMPK-P-Raptor-dependent inhibition of mTORC1 and genotoxic cell death.

\section{Additional files}

Additional file 1: Supplementary materials and methods. (PDF $153 \mathrm{~kb}$ )

Additional file 2: Table S1. (XLS $163 \mathrm{~kb}$ )

Additional file 3: Supplementary Figures and Table S2. (PDF 504 kb)

\section{Competing interests}

None of the authors have any financial or non-financial competing interests in the manuscript.

\section{Authors' contributions}

JJ designed the overall study and supervised the experiments. ARM performed most experiments and data analysis. ARM, LJS, MP, JJ designed and $A R M, M U, L S, M P, K P, P P, M Z$ and JJ prepared shRNA library. ARM, EL and JJ performed in vitro experiments; AS, ARM, EL and MM performed in vivo experiments; AMY and EA performed human tissue staining and interpreted the results. MET and DJK shared data from sequencing of TSC loci of SEGA-derived cells. KK, JB, WG and SJ diagnosed the patients, obtained clinical material and provided pathomorphological assessment. ARM prepared figures and jointly with J J wrote the manuscript. All authors read and approved the final manuscript.

\section{Acknowledgements}

We express our gratitude to Magdalena Blazejczyk, Agnieszka Chacinska, Jozef Dulak and Robert Erickson for their helpful comments on the manuscript. We thank Michal Wasilewski, Agnieszka Chacinska, Jozef Dulak and Artur Jozwik for contributing antibodies and their expertise, Piotr Stepniewski for help with live imaging data handling, and Phebe Wulf, Katarzyna Rydz, Alina Zielinska, and Monika Matuszczyk for technical assistance. We also express our gratitude to Dr. T. Kobayashi for providing Tsc2-encoding plasmids and Dr. Harold Cremer for the pCx EGFP-N1 plasmids. The research leading to these results received funding from the Polish Ministry of Science and Higher Education (grant no. PBZ-MNil-2/1/ 2005) and the European Community's Seventh Framework Programme (FP7/2007-2013; EPISTOP, grant agreement $n^{\circ}$ 602391). The work of AM was financed by a National Science Centre grant no. 2011/03/B/NZ3/01970 and within the Parent-Bridge program of the Foundation for Polish Science, co-financed by the European Union under the European Regional Development Fund. The work of EL was supported by ERA-NET NEURON (grant no. 06/2011 'AMRePACELL', co-financed by NCBiR), Homing Plus programme (HOMING PLUS/ 2012-5/6) of the Foundation for Polish Science, cofinanced from the European Union under the European Regional Development Fund and National Science Centre "Sonata" grant no. 2013/11/D/NZ3/01079. The work of MM was supported by National Science Centre grant "Opus" no. 2012/05/B/NZ3/ 00429. JJ and AM are also recipients of the Foundation for Polish Science "Mistrz" Professorial Subsidy and Fellowship, respectively.

\section{Author details}

${ }^{1}$ Laboratory of Molecular and Cellular Neurobiology, International Institute of Molecular and Cell Biology, Warsaw 02-109, Poland. '2Department of (Neuro)Pathology, Academic Medical Center, Amsterdam 1105 AZ, The Netherlands. ${ }^{3}$ Swammerdam Institute for Life Sciences and Center for Neuroscience, University of Amsterdam, Amsterdam 1090 GE, The Netherlands. ${ }^{4}$ Stichting Epilepsie Instellingen Nederland (SEIN), Heemstede 2103 SW, The Netherlands. 'Department of Neurology and Epileptology, Children's Memorial Health Institute, Warsaw 04-730, Poland. 'Division of Pulmonary Medicine and of Genetics, Brigham and Women's Hospital, Harvard Medical School, Boston, MA 02-115, USA. ${ }^{7}$ Department of Child Neurology, Medical University of Warsaw, 02-091 Warsaw, Poland.

Received: 11 July 2015 Accepted: 16 July 2015

Published online: 30 July 2015

\section{References}

1. Bailey HH, Mulcahy RT, Tutsch KD, Arzoomanian RZ, Alberti D, Tombes MB, Wilding G, Pomplun M, Spriggs DR (1994) Phase I clinical trial of intravenous L-buthionine sulfoximine and melphalan: an attempt at modulation of glutathione. J Clin Oncol 12:194-205 
2. Boutin C, Diestel S, Desoeuvre A, Tiveron M-C, Cremer H (2008) Efficient in vivo electroporation of the postnatal rodent forebrain. PLoS One 3:e1883. doi:10.1371/journal.pone.0001883

3. Brummelkamp TR, Bernards R, Agami R (2002) A system for stable expression of short interfering RNAs in mammalian cells. Science 296:550-553. doi:10.1126/science.1068999

4. Buccoliero AM, Franchi A, Castiglione F, Gheri CF, Mussa F, Giordano F, Genitori L, Taddei GL (2009) Subependymal giant cell astrocytoma (SEGA): Is it an astrocytoma? Morphological, immunohistochemical and ultrastructural study. Neuropathology 29:25-30. doi:10.1111/j.1440-1789.2008.00934.x

5. Butcher RA, Bhullar BS, Perlstein EO, Marsischky G, LaBaer J, Schreiber SL (2006) Microarray-based method for monitoring yeast overexpression strains reveals small-molecule targets in TOR pathway. Nat Chem Biol 2:103-109. doi:10.1038/nchembio762

6. Chan TF, Carvalho J, Riles L, Zheng X (2000) A chemical genomics approach toward understanding the global functions of the target of rapamycin protein (TOR). Proc Natl Acad Sci 97:13227

7. Drakos E, Atsaves V, Li J, Leventaki V, Andreeff M, Medeiros LJ, Rassidakis GZ (2009) Stabilization and activation of p53 downregulates mTOR signaling through AMPK in mantle cell lymphoma. Leukemia 23:784-790. doi:10.1038/leu.2008.348

8. Feliciano DM, Su T, Lopez J, Platel J-C, Bordey A (2011) Single-cell Tsc1 knockout during corticogenesis generates tuber-like lesions and reduces seizure threshold in mice. J Clin Invest 121:1596-1607. doi:10.1172/JCl44909

9. Feliciano DM, Zhang S, Quon JL, Bordey A (2013) Hypoxia-inducible factor 1a is a Tsc1-regulated survival factor in newborn neurons in tuberous sclerosis complex. Hum Mol Genet 22:1725-1734. doi:10.1093/hmg/ddt018

10. Feng Z, Zhang H, Levine AJ, Jin S (2005) The coordinate regulation of the p53 and mTOR pathways in cells. Proc Natl Acad Sci U S A 102:8204-8209. doi:10.1073/pnas.0502857102

11. Gomez MR (1999) Definition and criteria for diagnosis. In: Tuberous Scler. Complex, 3rd edn. Oxford, New York, pp 10-23

12. Goto J, Talos DM, Klein P, Qin W, Chekaluk YI, Anderl S, Malinowska IA, Di Nardo A, Bronson RT, Chan JA, Vinters HV, Kernie SG, Jensen FE, Sahin M, Kwiatkowski DJ (2011) Regulable neural progenitor-specific Tsc1 loss yields giant cells with organellar dysfunction in a model of tuberous sclerosis complex. Proc Natl Acad Sci U S A 108:E1070-1079. doi:10.1073/pnas.1106454108

13. Grajkowska W, Kotulska K, Jurkiewicz E, Matyja E (2010) Brain lesions in tuberous sclerosis complex. Review. Folia Neuropathol 48:139-149

14. Griffith OW (1982) Mechanism of action, metabolism, and toxicity of buthionine sulfoximine and its higher homologs, potent inhibitors of glutathione synthesis. J Biol Chem 257:13704-13712

15. Gwinn DM, Shackelford DB, Egan DF, Mihaylova MM, Mery A, Vasquez DS, Turk BE, Shaw RJ (2008) AMPK phosphorylation of raptor mediates a metabolic checkpoint. Mol Cell 30:214-226. doi:10.1016/j.molcel.2008.03.003

16. Ishii T, Itoh K, Takahashi S, Sato H, Yanagawa T, Katoh Y, Bannai S, Yamamoto M (2000) Transcription factor Nrf2 coordinately regulates a group of oxidative stress-inducible genes in macrophages. J Biol Chem 275:16023-16029. doi:10.1074/jbc.275.21.16023

17. Jain A, Martensson J, Stole E, Auld PA, Meister A (1991) Glutathione deficiency leads to mitochondrial damage in brain. Proc Natl Acad Sci U S A 88:1913-1917

18. Jansen FE, van Huffelen AC, Algra A, van Nieuwenhuizen O (2007) Epilepsy surgery in tuberous sclerosis: a systematic review. Epilepsia 48:1477-1484. doi:10.1111/j.1528-1167.2007.01117.x

19. Jaworski J, Spangler S, Seeburg DP, Hoogenraad CC, Sheng M (2005) Control of dendritic arborization by the phosphoinositide-3'-kinase-Aktmammalian target of rapamycin pathway. J Neurosci 25:11300-11312. doi:10.1523/JNEUROSCI.2270-05.2005

20. Kotulska K, Borkowska J, Jozwiak S (2013) Possible prevention of tuberous sclerosis complex lesions. Pediatrics 132:e239-242. doi:10.1542/peds. 2012-3607

21. Kotulska K, Chmielewski D, Borkowska J, Jurkiewicz E, Kuczyński D, Kmieć T, Łojszczyk B, Dunin-Wąsowicz D, Jóźwiak S (2013) Long-term effect of everolimus on epilepsy and growth in children under 3 years of age treated for subependymal giant cell astrocytoma associated with tuberous sclerosis complex. Eur J Paediatr Neurol 17:479-485. doi:10.1016/j.ejpn.2013.03.002

22. Lafourcade CA, Lin TV, Feliciano DM, Zhang L, Hsieh LS, Bordey A (2013) Rheb activation in subventricular zone progenitors leads to heterotopia, ectopic neuronal differentiation, and rapamycin-sensitive olfactory micronodules and dendrite hypertrophy of newborn neurons. J Neurosci 33:2419-2431. doi:10.1523/JNEUROSCI.1840-12.2013
23. Li J, Csibi A, Yang S, Hoffman GR, Li C, Zhang E, Yu JJ, Blenis J (2015) Synthetic lethality of combined glutaminase and Hsp90 inhibition in mTORC1-driven tumor cells. Proc Natl Acad Sci U S A 112:E21-29. doi:10.1073/pnas.1417015112

24. Louis DN, Ohgaki H, Wiestler OD, Cavenee WK, Burger PC, Jouvet A, Scheithauer BW, Kleihues P (2007) The 2007 WHO classification of tumours of the central nervous system. Acta Neuropathol (Berl) 114:97-109. doi:10.1007/s00401-007-0243-4

25. Malik AR, Urbanska M, Gozdz A, Swiech $\sqcup$, Nagalski A, Perycz M, Blazejczyk M, Jaworski J (2013) Cyr61, a matricellular protein, is needed for dendritic arborization of hippocampal neurons. J Biol Chem 288:8544-8559. doi:10.1074/jbc.M112.411629

26. Malik AR, Urbanska M, Macias M, Skalecka A, Jaworski J (2013) Beyond control of protein translation: what we have learned about the noncanonical regulation and function of mammalian target of rapamycin (mTOR). Biochim Biophys Acta 1834:1434-1448. doi:10.1016/ j.bbapap.2012.12.010

27. Mizuguchi M, Takashima S (2001) Neuropathology of tuberous sclerosis. Brain Dev 23:508-515

28. Di Nardo A, Kramvis I, Cho N, Sadowski A, Meikle L, Kwiatkowski DJ, Sahin M (2009) Tuberous sclerosis complex activity is required to control neuronal stress responses in an mTOR-dependent manner. J Neurosci 29:5926-5937. doi:10.1523/JNEUROSCI.0778-09.2009

29. Orlova KA, Crino PB (2010) The tuberous sclerosis complex. Ann N Y Acad Sci 1184:87-105. doi:10.1111/j.1749-6632.2009.05117.x

30. Ouyang T, Zhang N, Benjamin T, Wang L, Jiao J, Zhao Y, Chen J (2014) Subependymal giant cell astrocytoma: current concepts, management, and future directions. Childs Nerv Syst. doi:10.1007/s00381-014-2383-x

31. Ouyang T, Zhang N, Benjamin T, Wang L, Jiao J, Zhao Y, Chen J (2014) Subependymal giant cell astrocytoma: current concepts, management, and future directions. Childs Nerv Syst 30:561-570. doi:10.1007/s00381-014-2383-x

32. Ozcan U, Ozcan L, Yilmaz E, Düvel K, Sahin M, Manning BD, Hotamisligil GS (2008) Loss of the tuberous sclerosis complex tumor suppressors triggers the unfolded protein response to regulate insulin signaling and apoptosis. Mol Cell 29:541-551. doi:10.1016/j.molcel.2007.12.023

33. Parker WE, Orlova KA, Heuer GG, Baybis M, Aronica E, Frost M, Wong M, Crino PB (2011) Enhanced epidermal growth factor, hepatocyte growth factor, and vascular endothelial growth factor expression in tuberous sclerosis complex. Am J Pathol 178:296-305. doi:10.1016/j.ajpath.2010.11.031

34. Perek-Polnik M, Jóźwiak S, Jurkiewicz E, Perek D, Kotulska K (2012) Effective everolimus treatment of inoperable, life-threatening subependymal giant cell astrocytoma and intractable epilepsy in a patient with tuberous sclerosis complex. Eur J Paediatr Neurol 16:83-85. doi:10.1016/ j.ejpn.2011.09.006

35. Perycz M, Urbanska AS, Krawczyk PS, Parobczak K, Jaworski J (2011) Zipcode binding protein 1 regulates the development of dendritic arbors in hippocampal neurons. J Neurosci 31:5271-5285. doi:10.1523/ JNEUROSCI.2387-10.2011

36. Prabowo AS, Anink JJ, Lammens M, Nellist M, van den Ouweland AMW, Adle-Biassette H, Sarnat HB, Flores-Sarnat L, Crino PB, Aronica E (2013) Fetal brain lesions in tuberous sclerosis complex: TORC1 activation and inflammation. Brain Pathol 23:45-59. doi:10.1111/j.1750-3639.2012.00616.x

37. Shieh SY, Ikeda M, Taya Y, Prives C (1997) DNA damage-induced phosphorylation of p53 alleviates inhibition by MDM2. Cell 91:325-334

38. Somers MJG, Paul E (2014) Safety considerations of mammalian target of rapamycin inhibitors in tuberous sclerosis complex and renal transplantation. J Clin Pharmacol. doi: 10.1002/jcph.428

39. Tavazoie SF, Alvarez VA, Ridenour DA, Kwiatkowski DJ, Sabatini BL (2005) Regulation of neuronal morphology and function by the tumor suppressors Tsc1 and Tsc2. Nat Neurosci 8:1727-1734. doi:10.1038/nn1566

40. Trachootham D, Alexandre J, Huang P (2009) Targeting cancer cells by ROS-mediated mechanisms: a radical therapeutic approach? Nat Rev Drug Discov 8:579-591. doi:10.1038/nrd2803

41. Trelinska J, Dachowska I, Kotulska K, Fendler W, Jozwiak S, Mlynarski W (2015) Complications of mammalian target of rapamycin inhibitor anticancer treatment among patients with tuberous sclerosis complex are common and occasionally life-threatening. Anticancer Drugs 26:437-442. doi:10.1097/CAD.0000000000000207

42. Tyburczy ME, Kotulska K, Pokarowski P, Mieczkowski J, Kucharska J, Grajkowska W, Roszkowski M, Jozwiak S, Kaminska B (2010) Novel proteins regulated by $\mathrm{mTOR}$ in subependymal giant cell astrocytomas of patients with tuberous sclerosis complex and new therapeutic implications. Am J Pathol 176:1878-1890. doi:10.2353/ajpath.2010.090950 
43. Way SW, McKenna J 3rd, Mietzsch U, Reith RM, Wu HC-J, Gambello MJ (2009) Loss of Tsc2 in radial glia models the brain pathology of tuberous sclerosis complex in the mouse. Hum Mol Genet 18:1252-1265. do: $10.1093 / \mathrm{hmg} / \mathrm{ddp} 025$

44. Wiegand G, May TW, Ostertag P, Boor R, Stephani U, Franz DN (2013) Everolimus in tuberous sclerosis patients with intractable epilepsy: a treatment option? Eur J Paediatr Neurol EJPN Off J Eur Paediatr Neurol Soc 17:631-638. doi:10.1016/j.ejpn.2013.06.002

45. Xie MW, Jin F, Hwang H, Hwang S, Anand V, Duncan MC, Huang J (2005) Insights into TOR function and rapamycin response: chemical genomic profiling by using a high-density cell array method. Proc Natl Acad Sci U S A 102:7215-7220. doi:10.1073/pnas.0500297102

\section{Submit your next manuscript to BioMed Central and take full advantage of:}

- Convenient online submission

- Thorough peer review

- No space constraints or color figure charges

- Immediate publication on acceptance

- Inclusion in PubMed, CAS, Scopus and Google Scholar

- Research which is freely available for redistribution 